\title{
Spontaneous Intracranial Hypotension: An Unusual Debut and An
}

\section{Exceptional Evolution}

\section{Cristina del Toro-Pérez | Pedro Guardado Santervás | Patricia Martínez-Sánchez* | Antonio Arjona- Padillo}

*Correspondence: Patricia Martínez Sánchez

Address: Neurosonology Laboratory, Department of Neurology, Torrecárdenas University Hospital, University of Almería, Spain e-mail $\bowtie$ : patrinda@ual.es

Received: 05 November 2021; Accepted: 15 November 2021

Copyright: (C) 2021 del Toro-Pérez C. This is an open-access article distributed under the terms of the Creative Commons Attribution License, which permits unrestricted use, distribution, and reproduction in any medium, provided that the original work is properly cited.

\section{ABSTRACT}

Spontaneous intracranial hypotension (SIH) is an entity that requires a high level of suspicion in order to request specific tests for its detection. However, its wide clinical variability can make it difficult to diagnose. The objective of this case report is to review several features of SIH through two atypical clinical cases; the first one with an atypical debut and the second one with an unexpected clinical evolution.

The first patient presents with a focal seizure due to cerebral venous sinus thrombosis without headache attributable to hypotension, being later diagnosed following neuroimaging. The second patient presents with a characteristic picture of SIH with initial response to treatment. However, he undergoes an unusual subsequent evolution with the final diagnosis of Primary Central Nervous System Lymphoma, which makes it necessary to consider this disease as a possible etiology of SIH, a relationship not described to date in the literature.

The two atypical cases of SIH demonstrate that thanks to a better understanding of this entity it is possible to diagnose patients without the classic clinical presentation. Once the diagnosis is established, it is important to be alert to the possibility of an unusual clinical course.

Keywords: Intracranial Hypotension, Cerebrospinal Liquid Leak, Epilepsy, Headache, Nausea, Orthostatic Spontaneous, Subdural Hematoma, Thrombosis, Primary Central Nervous System Lymphoma, Case report

\section{Introduction}

A syndrome similar to lumbar puncture headache was first described in 1939 (Mokri 2013). This syndrome also consisted of an orthostatic headache and low cerebrospinal fluid (CSF) opening pressure, but unlike post-lumbar puncture headache, this syndrome was spontaneous. Years later, this syndrome was termed Spontaneous Intracranial Hypotension (SIH). Since then, numerous case series have been published, most of them following the description of its typical findings on MRI (Mokri, 2013; Krantz et al., 2018). In recent decades there has been an increase in the number of published cases due to a better understanding of this disease, which can be self-limiting with conservative treatment (Mokri, 2013). In a 
recent systematic review of this entity, orthostatic headache was found in $87-96 \%$ of cases and $3 \%$ had no headache at all (D'Antona l et al., 2021). Patients may present with a long list of associated or isolated symptoms (Mokri, 2013; Krantz et al., 2018) that also include multiple complications (Table 1).

Table 1: Complications in Spontaneous Intracranial Hypotension (Mokri, 2013; Daroff et al., 2016; Garcia-Carreira et al., 2014)

\begin{tabular}{|l|}
\hline Subdural hematomas and hygromas \\
\hline Rebound intracranial hypertension \\
\hline Cerebral venous sinuses thrombosis \\
\hline Central herniation \\
\hline Bibrachial amyotrophy \\
\hline Superficial siderosis \\
\hline Syringomyelia \\
\hline lschemic stroke \\
\hline Frontotemporal syndrome \\
\hline Ataxia, parkinsonism and bulbar dysfunction \\
\hline Hyperprolactinemia \\
\hline
\end{tabular}

We present two cases of HIS: one patient debuts with an atypical clinical onset and the second patient presents an unexpected evolution and complications, showing the complexity of this disease.

\section{Case Report}

Patients underwent a detailed physical examination, cranial CT, brain MRI with gadolinium and finally 111In-diethylenetriaminepentaacetic acid (111In-DTPA) cisternography after suspicion of SIH. Based on the results of brain MRI and isotopic cisternography, the patients were diagnosed with SIH. None of them reported previous trauma. Main neuroimaging findings are shown in Figure 1.

\section{Case 1: Clinical Onset with Focal Seizure Secondary to Cerebral Venous Sinus Thrombosis (CVST)} Without Orthostatic Headache

A 62-year-old woman with vertigo and episodic migraine was admitted to Neurology Department after a first focal seizure with secondary generalization preceded by nausea, vomiting, diarrhea and a predominantly nocturnal headache with drowsiness of several days of evolution. Prior to these symptoms, she refers a self-limited high dorsal pain after moderate physical exertion. Cerebral venous sinus thrombosis (CVST) was diagnosed by brain MRI with venous angioRM and the patient was discharged asymptomatic after anticoagulation with acenocoumarol. Some tests to complete the etiological study were pending at the discharge. Signs of SIH in control brain MRI were observed in hospital outpatient consultations, so the patient was admitted again on a scheduled basis to try to identify a possible CSF leak. 


\section{Complementary Tests}

Blood tests were normal or negative (including autoimmunity and complete thrombophilia). Normal brain CT. Brain MRI with venous MRI angiography (three days after lumbar puncture): hyperintensity inside the right jugular vein, filling defect in the right lateral sinus and superior longitudinal sinus, with diffuse enhancement of the dura after contrast administration. Analysis of CSF obtained by lumbar puncture (LP): 2 leukocytes (corrected for blood cells) with normal protein and glucose and normal opening pressure (15-16 cm of water).

Control brain MRI showed dural thickening with meningeal enhancement and CSF collection in the subdural space corresponding to a hygroma, as well as resolution of venous sinus thrombosis.

CNS 111In-DTPA cisternography, performed five months after the first admission, revealed multiple heterotopic tracer deposits suggestive of CSF leakage into the extraspinal space at the bilateral thoracic level.

\section{Diagnosis}

SIH secondary to idiopathic CSF leak at dorsal spinal level without orthostatic headache. Jugular SVT secondary to intracranial hypovolemia. Focal seizures with generalization secondary to CSVT.

\section{Treatment and Evolution}

An autologous blood patch was performed. Three months later, radiological signs of intracranial hypotension with meningeal enhancement in D11 persisted in the control brain MRI. A third admission for a new autologous patch was performed and the subsequent evolution was favorable, with resolution of the radiological findings, remaining clinically asymptomatic at sixty months.

Case 2: Clinical Presentation with Orthostatic Headache and Subdural Hematoma with Subsequent Finding of Primary Central Nervous System (CNS) Lymphoma

A 59-year-old man consulted the emergency department (ED) for moderate headache, predominantly in the morning, which appeared when standing upright and improved when lying down, accompanied by daytime somnolence, photo and sonophobia. He was diagnosed with SIH possibly related to lumbar osteophytes based on cranial and spinal MRI and isotopic cisternography findings. He required treatment with epidural autologous blood patch due to decreased level of consciousness, with excellent initial response. After discharge she had a torpid evolution with subdural hygroma-hematoma that required surgical intervention, where a sample of the dura mater was taken for study. He consulted 
during the following month for focal motor crises and Neurosurgery prescribed antiepileptic treatment with Levetiracetam and oral corticosteroids (twenty days).

Finally, he was readmitted to neurology for clinical worsening with post-surgical pneumocephaly, somnolence and persistence of focal motor seizures with subsequent status epilepticus.

\section{Complementary Tests}

Laboratory tests in the ED and during the stay in Neurology showed no abnormalities. The cranial CT scan performed in the ED showed bilateral thick frontoparietal collections with a density similar to that of CSF, suggestive of bilateral hygroma/subdural hygroma. Brain MRI with and without contrast: two bilateral homogeneous supratentorial subdural collections were observed, hypointense in T1 and hyperintense in T2 and FLAIR; an homogeneous and slight contrast enhancement of the dura mater was observed, extending through both cerebral hemispheres. A thickened and enhanced pituitary gland and distended dural sinuses were also observed. The midbrain was descended and the lateral ventricles were small. Cervical, dorsal and lumbosacral MRI: there was a loss of height of the L4-L5 intervertebral disc with osteophytes and marked hypertrophy of the frontal joints leading to compression of the dural sac. Isotopic cisternography two months after admission: at 24 hours there was slight activity in both hemispheres and little activity on the cerebral convexities.

Simple cranial CT, three months after the first admission, showed an extensive hypodense right frontotemporal area, with associated edema and generalized sulcus effacement. EEG showed a generalized non-convulsive state. Post-surgical cranial CT scan: right frontal pneumocephaly. Pathological study of the dura sample: activated lymphocytes. Brain MRI: cerebral edema and bilateral subdural collections. Diffuse thickening and soft enhancement of the dura. T2-weighted MRI and FLAIR showed hyperintensities in the left frontal lobe and in the knee of the corpus callosum. CSF three months after the first admission was normal: biochemistry, cytology (3 samples) and opening pressure. Brain biopsy: presence of histiocytic infiltrate, T lymphocytes and increased glial cellularity of gemistocytic appearance of apparent reactive nature. Perivascular infiltration of atypical B lymphocytes and Bcl2 positive, enhanced by histochemical technique for reticulin.

\section{Diagnosis}

SIH secondary to non-localized leak. Chronic subdural hygromas-hematomas symptomatic to CSF hypotension. Post-surgical pneumocephaly. Primary CNS lymphoma. 


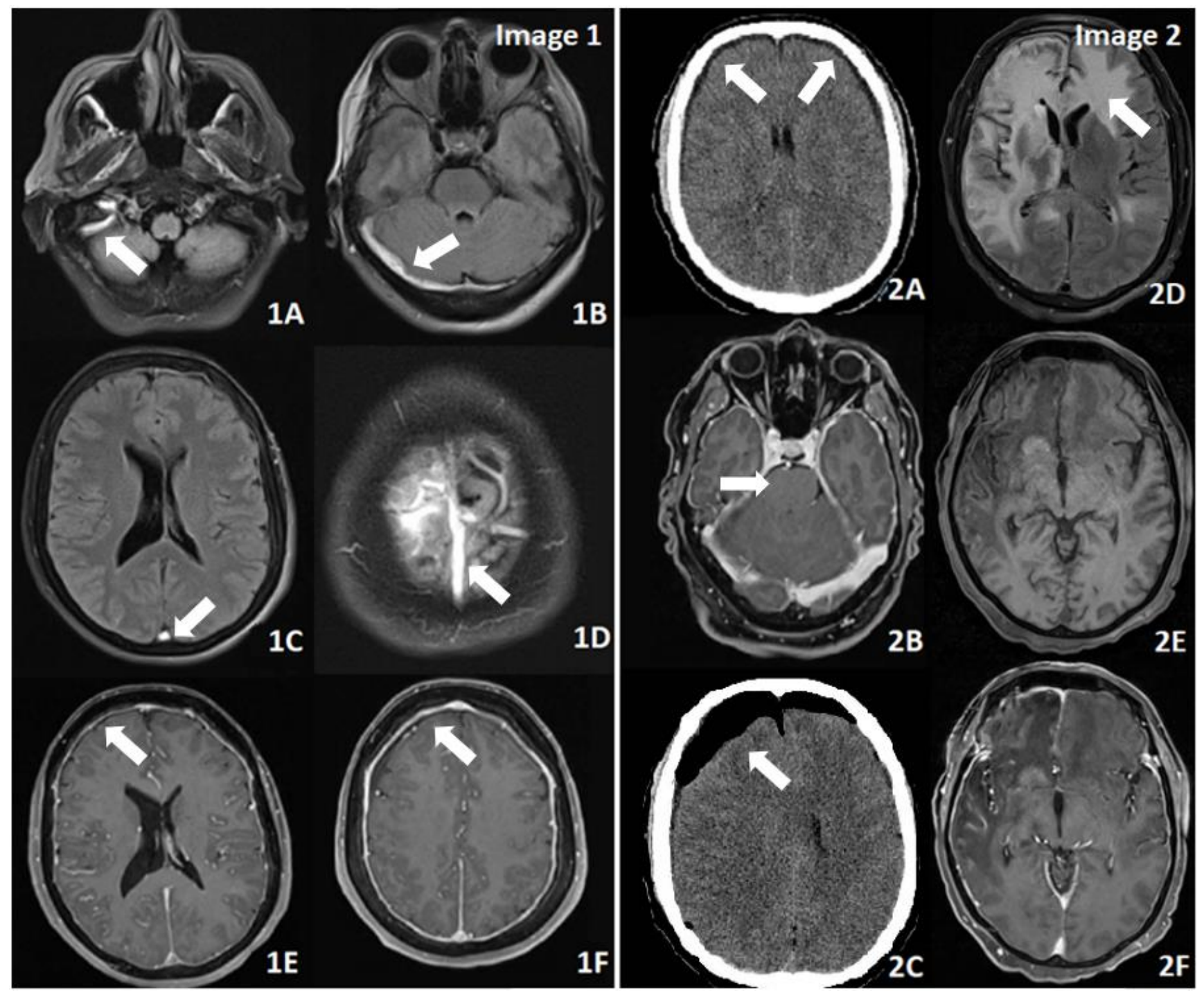

Figure 1: Imaging findings in SIH (white arrows). Brain MRI with contrast Case 1. 1A, 1B, 1C, 1D show thrombosis of the right jugular vein, right lateral and superior longitudinal sinus, respectively. In $1 \mathrm{E}$ and $1 \mathrm{~F}$, pachymeningeal enhancement is seen after contrast. Image 2. Case 2. 2A. Brain CT showed bilateral hygromas from the onset of the clinical picture, with partial collapse of the ventricular system. 2B. Anteroposterior elongation of the brainstem. 2C. Pneumoencephaly as a postsurgical complication. 2D, 2E, 2F. FLAIR, T1 without contrast and T1 with contrast sequences of the brain show cerebral edema with involvement of the genu of the corpus callosum. A biopsy of the lesion was performed, with the final diagnosis of Primary Central Nervous System Lymphoma.

\section{Treatment and Evolution}

During the second admission, the patient had a torpid evolution despite treatment with dexamethasone prescribed for edema caused by subdural collections. The cerebral edema worsened on MRI and a contralateral white matter lesion with involvement of the genu of the corpus callosum was also observed. Accordingly, a biopsy was performed with a result of primary CNS lymphoma. Conservative 
treatment was agreed with his wife. The patient died with adequate palliative treatment after five months.

\section{Discussion}

Our contribution are two unusual cases: one with a debut of venous sinus thrombosis without orthostatic headache and the second exceptional case with the classic clinical debut and its surprising evolution to a primary cerebral lymphoma, which inevitably leads us to relate both disorders.

The occurrence of CVST (Case 1) is widely described as a complication of SIH and occurs in approximately $1 \%$ of cases (Zhang D et al., 2018), secondary to venodilatation and venous stasis according to the Monro-Kellie doctrine (Mokri, 2013). The initial symptoms in this patient can be attributed to venous thrombosis with symptomatic focal crisis onset and nonspecific headache of short duration. The patient had no orthostatic headache or other SIH-related symptoms at any time, which hampered the diagnosis. The diagnosis was made on the basis of typical findings on gadolinium MRI, although this was performed after a lumbar puncture, which added another diagnostic challenge.

There is sometimes doubt that a diagnostic lumbar puncture could have caused the findings that characterize SIH on cranial MRI. Although this option is possible, its presence is rare and in one study explained only $1.2 \%$ of radiological findings after lumbar puncture (Wesley SF et al., 2016). In our case, isotopic cisternography was subsequently performed with multiple leaks at the spinal level, confirming the diagnosis.

Finally, the second case debuted with classic symptoms, brain MRI findings and isotopic cisternography of the SIH. Initial and transient resolution was obtained with an epidural patch, also compatible with a SIH. Subsequently the patient suffered a torpid evolution with the final demonstration of a primary CNS lymphoma adjacent to the operated area. Due to the classic findings in the tests performed we cannot attribute the dural thickening to lymphoma. Moreover, the pattern of lymphomatous enhancement is usually leptomeningeal or mixed (Wesley SF et al., 2016). We have not found any similar description in the literature. Our hypothesis to explain a relationship between the two is the existence of a subclinical lymphoma as a cause of dural weakness. This would cause a predisposition to a SIH or a modification in the biological behavior of the subclinical lymphoma after presenting an important intercurrent process, treated for a few weeks with oral corticosteroids.

\section{Conclusion}

We present two atypical clinical cases of SIH that demonstrate, on the one hand, that it is possible to 
diagnose this entity in patients who do not have orthostatic headache or low CSF pressure, and on the other hand, that once the diagnosis is established, we must be attentive to its evolution and eventual complications.

Ethical Approval: The authors declare no conflict of interest. Therapeutic clinical decisions were supported by the best available evidence from health care research.

Written Informed Consent for Publication of Case: Written informed consent was obtained from Case 1 patient for publication of this case report and any accompanying images. No familiar of Case 2 patient was found.

\section{References}

D'Antona L, Jaime Merchan MA, Vassiliou A, Watkins LD, Davagnanam I, Toma AK, Matharu MS. Clinical Presentation, Investigation Findings, and Treatment Outcomes of Spontaneous Intracranial Hypotension Syndrome A Systematic Review and Meta-analysis. JAMA Neurol 2021; 4: e204799.

Daroff R, Jankovic J, Mazziotta J, Pomeroy S. Neurology in Clinical Practice. 7th Ed. China: Elsevier, 2016.

Garcia-Carreira MC, Vergé DC, Branera J, Zauner M, Herrero JE, Tió E, Perpinyà GR. Cerebral Venous Thrombosis in Two Patients with Spontaneous Intracranial Hypotension. Case Rep Neurol Med 2014.

Krantz PG, Gray L, Amrhein TJ. Spontaneous Intracranial Hypotension: 10 Myths and Misperceptions. Headache 2018; 58: 948-959.

Mokri B (2013) Spontaneous Low Pressure, Low CSF Volume Headaches: Spontaneous CSF Leaks. Headache 2013; 53: 1034-1053

Smirniotopoulos JG, Murphy FM, Rushing EJ, Rees JH, Schroeder JW. Patterns of contrast enhancement in the brain and meninges. Radiographics 2007; 27: 525-551

Wesley SF, Garcia-Santibanez R, Liang J, Pyburn D. Incidence of meningeal enhancement on brain MRI secondary to lumbar puncture. Neurol Clin Pract 2016; 6: 315-320

Zhang D, Wang J, Zhang Q, He F, Hu X. Cerebral Venous Thrombosis in Spontaneous Intracranial Hypotension: A Report on 4 Cases and a Review of the Literature. Headache 2018; 58: 1244-1255. 